\title{
Intravenous hydroxocobalamin and crystal nephropathy
}

\section{Matthieu Legrand and Vincent Mallet}

We read with great interest the excellent review by S. R. Mulay and H.-J. Anders on crystal nephropathies (Crystal nephropathies: mechanisms of crystal-induced kidney injury. Nat. Rev. Nephrol. 13, 226-240; 2017) $)^{1}$. The authors list vitamin $\mathrm{C}$ as a cause of oxalate nephropathy, but do not mention the potential role of vitamin $B_{12}$ in this disease.

We recently reported cases of oxalate nephropathy after administration of the injectable form of vitamin $B_{12}$, hydroxocobalamin, in critically ill patients with burns and smoke inhalation injury ${ }^{2}$. The FDA has approved hydroxocobalamin for the treatment of cyanide poisoning. This agent chelates cyanide to form cyanocobalamin, which is excreted by the kidney.

We found that hydroxocobalamin administration was associated with a significant risk of acute kidney injury (AKI; OR 5.8,
95\% CI 1.6-20.7) in 99 critically ill patients with burns. In addition, evidence of oxalate nephropathy was present in kidney biopsy samples from two patients. We also observed oxaluria after hydroxocobalamin administration in patients who received this agent $(n=6)$ compared to the control group $(n=9)$. Interestingly, oxaluria was previously reported in healthy volunteers and animals that received hydroxocobalamin ${ }^{3}$.

The mechanism of AKI development in patients with burns might involve low tubular fluid flow owing to the severe dehydration that is observed in the initial phase after burn injury; this low flow might promote tubular deposits of oxalate crystals. The pathophysiology of oxalate nephropathy after vitamin $\mathrm{B}_{12}$ administration remains unexplored but might involve the methylmalonyl coenzyme A. Vitamin $\mathrm{B}_{12}$ acts as a cofactor of methylmalonyl-CoA mutase, which converts methylmalonyl CoA into succinyl CoA, which has a role in the metabolism of oxalate. This hypothesis requires confirmation. In the meantime, physicians should be aware of the link between oxalate generation and vitamin $B_{12}$ metabolism and the potential risk of oxalate nephropathy in patients receiving hydroxycobalamin.

Matthieu Legrand is at AP-HP, Hôpital St-Louis, Department of Anesthesiology and Critical Care and Burn Unit 1 Avenue Claude Vellefaux, 75010, Paris,

France.

Vincent Mallet is at AP-HP, Hepatology, Hôpital Cochin, 27 Rue du Faubourg Saint-Jacques, 75014 Paris, France.

Correspondence to M.L. matthieu.legrand@aphp.fr

doi:10.1038/nrneph.2017.108 Published online 31 Jul 2017

1. Mulay, S. R. \& Anders, H.-J. Crystal nephropathies: mechanisms of crystal-induced kidney injury. Nat. Rev. Nephrol. 13, 226-240 (2017).

2. Legrand, M. et al. Risk of oxalate nephropathy with the use of cyanide antidote hydroxocobalamin in critically ill burn patients. Intensive Care Med. $\mathbf{4 2}$ 1080-1081 (2016)

3. EMD Serono, A Division of EMD Inc., Canada. CYANOKIT® Product Monograph https://www.inspq. qc.ca/sites/default/files/toxicologie-clinique/bit/ hydroxocobalamine emd 20101217.pdf (2010).

Competing interests statement

The authors declare no competing interests. 